This item was submitted to Loughborough's Research Repository by the author.

Items in Figshare are protected by copyright, with all rights reserved, unless otherwise indicated.

\title{
The invariant functions and invariant-image conditions of the rational Bézier
} triangles

PLEASE CITE THE PUBLISHED VERSION

http://dx.doi.org/10.1007/s00200-012-0174-8

PUBLISHER

(C) Springer

VERSION

AM (Accepted Manuscript)

LICENCE

CC BY-NC-ND 4.0

REPOSITORY RECORD

Bez, Helmut E.. 2019. "The Invariant Functions and Invariant-image Conditions of the Rational Bézier Triangles”. figshare. https://hdl.handle.net/2134/14693. 
This item was submitted to Loughborough's Institutional Repository (https://dspace.lboro.ac.uk/) by the author and is made available under the following Creative Commons Licence conditions.

\section{creative
commons}

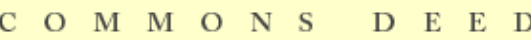

Attribution-NonCommercial-NoDerivs 2.5

You are free:

- to copy, distribute, display, and perform the work

Under the following conditions:

Attribution. You must attribute the work in the manner specified b the author or licensor.

Noncommercial. You may not use this work for commercial purposes.

No Derivative Works. You may not alter, transform, or build upon this work.

- For any reuse or distribution, you must make clear to others the license terms of this work.

- Any of these conditions can be waived if you get permission from the copyright holder.

Your fair use and other rights are in no way affected by the above.

This is a human-readable summary of the Leqal Code (the full license).

\section{Disclaimer 만}

For the full text of this licence, please go to: http://creativecommons.org/licenses/by-nc-nd/2.5/ 


\title{
The invariant functions and invariant-image conditions of the rational Bézier triangles
}

H.E. Bez

Received: date / Accepted: date

\begin{abstract}
A functional equation for invariant-image is presented and solved to identify the largest group of image-preserving weight transformations for the rational Bézier triangles. A fundamental theorem on the invariants of Lie group actions is used to demonstrate that $\frac{1}{2}(n+1)(n+2)-3$ real-valued, functionally-independent invariants exist for the degree $n$ triangles. Explicit forms of the real-valued invariants are derived; and a further, discrete-valued, invariant is identified and shown to complete the set required for invariant image.
\end{abstract}

Keywords Rational Parametrisation · Geometric Modeling · Transformation Groups · Invariant Functions

Mathematics Subject Classification (2000) 15A72 $26 \mathrm{C} 15 \cdot 34 \mathrm{C} 14 \cdot 35 \mathrm{~B} 06$.

$54 \mathrm{H} 15 \cdot 56 \mathrm{D} 17 \cdot 65 \mathrm{D} 18 \cdot 97 \mathrm{M} 50$

\section{Introduction}

Patterson [1] showed that if the weight vector $\omega=\left(\omega_{0}, \ldots, \omega_{n}\right)$ of a degree- $n$ Bézier rational path is transformed to $\omega^{*}=\left(\omega_{0}^{*}, \ldots, \omega_{n}^{*}\right)$, where $\omega_{i}>0$ and $\omega_{i}^{*}>0$, then the shape of the path is unchanged if:

$$
\varsigma_{i}\left(\omega^{*}\right)=\varsigma_{i}(\omega) \text { for all } 1 \leq i \leq n-1
$$

where $\varsigma_{i}(\omega)=\frac{\omega_{i-1} \omega_{i+1}}{\omega_{i}^{2}}$. The $n-1$ functions $\varsigma_{i}$ therefore determine invariant-image conditions for Bézier paths with strictly positive weights. Additionally invariants:

- occur in the computation of generic weight-vector normalisations,

- can provide information about the underlying curve type [2],

H.E. Bez

Department of Computer Science,

Loughborough University,

Loughborough, Leicestershire LE11 3TU.

Tel.: + $44(0) 1509222675$

Fax: + $44(0) 1509211586$

E-mail: h.e.bez@lboro.ac.uk 
- occur in the determination of optimum parametrisation with respect to a min-max condition on parametric 'speed' [3], [4].

Invariants play similar roles for Bézier surfaces, and recent work of the author has extended Patterson's results to the rational, rectangular biquadratic and bicubic Bézier surface parametrisations with arbitrary non-zero weights [5], [6]. Bézier parametrisations have the convex hull and other special properties in the case of all positive weights, hence negative weights are often not considered. However they may be difficult to avoid when it is required to parametrise surfaces with a small number of low-degree patches - in addition certain weight-sign changes are strongly coupled to the parametrisation of complementary regions of Bézier parametrisations [7], [8].

This paper focusses on the determination of the invariant functions and the invariantimage conditions of the rational Bézier triangles of arbitrary degree and with arbitrary non-zero weights. It is shown that $\frac{1}{2}(n+1)(n+2)-3$ real-valued invariants, and one discrete-valued invariant determine a complete set the triangles of degree $n$.

The approach expresses the image-invariance conditions for the Bézier triangles in a functional equation - the solution of which determines the largest transformation group of the weights compatible with invariant image. The fundamental theorem on invariants of Lie groups is then applied to demonstrate that $\frac{1}{2}(n+1)(n+2)-3$ functionally-independent, real-valued invariants exist for the degree- $n$ triangles. Using methods similar to those developed for the biquadratic and bicubic Bézier functions, the explicit form of a complete set of real-valued invariants is computed and shown, when augmented by a single discrete-valued invariant, to determine a complete set.

\section{The rational Bézier triangles in summation-free form}

The parametric domain of the triangular Bézier surfaces is the triangular subset, $\Delta=$ $\{(t, s): t>0, s>0, t+s \leq 1\}$, of $[0,1] \times[0,1]$. The rational, degree- $n$ triangular patch is defined for $(t, s) \in \Delta$ by:

$$
\tau_{B}[v, \omega](t, s)=\frac{\sum_{i+j+k=n} \frac{n !}{i ! j ! k !}(1-t-s)^{i} t^{j} s^{k} \omega_{i j k} v_{i j k}}{\sum_{i+j+k=n} \frac{n !}{i ! j ! k !}(1-t-s)^{i} t^{j} s^{k} \omega_{i j k}}
$$

on the vertices $\left\{v_{n 00}, \ldots, v_{0 n 0}\right\}, v_{i j k} \in \mathbb{R}^{3}$, and weights $\left\{\omega_{n 00}, \ldots, \omega_{0 n 0}\right\}, \omega_{i j k} \neq 0$. Hence $\tau_{B}$ may be regarded as a function-valued function on the domain $V_{n} \times \mathcal{O}_{n}$, where $V_{n}$ is the set of all $\frac{1}{2}(n+1)(n+2)-3$-tuples of vectors in $\mathbb{R}^{3}$ and $\mathcal{O}_{n}$ is the set of all $\frac{1}{2}(n+1)(n+2)$-tuples of non-zero real numbers.

The rational rectangular Bézier surfaces of degree $(n, n)$ may be expressed as linear combinations of the tensor-product basis functions $\mathrm{t} \otimes \mathrm{s}=\left[1, t, \ldots, t^{n}\right] \otimes\left[1, s, \ldots, s^{n}\right]$. The basis functions for the triangles are not of tensor-product form, however they may be expressed as linear combinations of the $\frac{(n+1)(n+2)}{2}$-element subset

$$
\pi_{n}(\mathrm{t} \otimes \mathrm{s})=\left[1, s, s^{2}, \ldots, s^{n} ; t, t s, t s^{2}, \ldots, t s^{n-1} ; t^{2}, t^{2} s, \ldots, t^{2} s^{n-2} ; \ldots, t^{n-1}, t^{n-1} s, t^{n}\right]
$$

of $\mathrm{t} \otimes \mathrm{s}$. In terms of $\pi_{n}(\mathrm{t} \otimes \mathrm{s})$ the rational degree- $n$ Bézier triangles may be written in summation-free form as:

$$
\tau_{B}[v, \omega](t, s)=\frac{\pi_{n}(\mathrm{t} \otimes \mathrm{s}) T_{n} \Omega_{n} v}{\pi_{n}(\mathrm{t} \otimes \mathrm{s}) T_{n} \omega}
$$

where 
$-T_{n}$ is a $\frac{(n+1)(n+2)}{2} \times \frac{(n+1)(n+2)}{2}$ upper triangular, 'structure' matrix for the degree- $n$ triangles

$-\Omega_{n}=\operatorname{diag}\left(\omega_{n 00}, \omega_{n-101}, \ldots, \omega_{00 n} ; \omega_{n-110}, \ldots, \omega_{01 n-1} ; \omega_{n-220}, \ldots, \omega_{0 n-22} ; \omega_{1 n-10}, \omega_{0 n-11}, \omega_{0 n 0}\right)$

$-v=\left(v_{n 00}, v_{n-101}, \ldots, v_{00 n} ; v_{n-110}, \ldots, v_{01 n-1} ; v_{n-220}, \ldots, v_{0 n-22} ; v_{1 n-10}, v_{0 n-11}, v_{0 n 0}\right)^{T}$

$-\omega=\left(\omega_{n 00}, \omega_{n-101}, \ldots, \omega_{00 n} ; \omega_{n-110}, \ldots, \omega_{01 n-1} ; \omega_{n-220}, \ldots, \omega_{0 n-22} ; \omega_{1 n-10}, \omega_{0 n-11}, \omega_{0 n 0}\right)^{T}$.

It will be seen later that, for the computations of this paper, it is sufficient if only the last three columns of the structure matrix are known. It is easy to show that

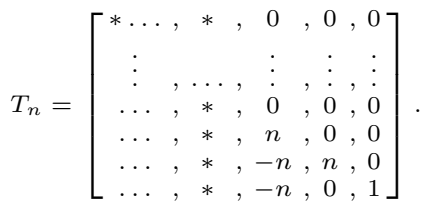

The explicit form of the structure matrices for the triangles of up to degree 3 are given in the appendix to the paper.

\section{The invariant-image functional equation}

The weight vectors $\omega, \omega^{*} \in \mathcal{O}_{n}$ determine the same image under $\tau_{B}$, for all $v \in V_{n}$, if and only if there exists a re-parametrisation function $\psi: \Delta \rightarrow \Delta$ such that

$$
\tau_{B}\left[v, \omega^{*}\right]=\tau_{B}[v, \omega] \circ \psi \text { for all } v \in V_{n} .
$$

The general solution of this functional equation, comprising all $\psi$ functions and $\omega^{*}$ vectors as functions of $\omega$, determines the relationships between the weight vectors $\omega$ and $\omega^{*}$ for constant geometry on any vertex set $v$ - relation (3) may therefore be referred to as the invariant-image functional equation for the Bézier triangles. All reparametrisation functions $\psi$ may be written as $\psi=(\phi, \mu)$.

In terms of the summation-free representation, relation (3) may be expressed as:

$$
\frac{\pi(\mathrm{t} \otimes \mathrm{s}) T_{n} \Omega_{n}^{*} v}{\pi(\mathrm{t} \otimes \mathrm{s}) T_{n} \omega^{*}}=\frac{\pi(\phi \otimes \mu) T_{n} \Omega_{n} v}{\pi(\phi \otimes \mu) T_{n} \omega}
$$

and, as this is required to hold for all $v$, it may be reduced to:

$$
\frac{\pi(\mathrm{t} \otimes \mathrm{s}) T_{n} \Omega_{n}^{*}}{\pi(\mathrm{t} \otimes \mathrm{s}) T_{n} \omega^{*}}=\frac{\pi(\phi \otimes \mu) T_{n} \Omega_{n}}{\pi(\phi \otimes \mu) T_{n} \omega} .
$$

\section{The functional solutions}

Lemma 1 The only solutions of the functional equation (4) are:

$$
\begin{aligned}
\phi(t, s) & =\frac{a t}{(1-t-s)+a t+b s} \\
\text { and } \quad & r(t, s)=\frac{b s}{(1-t-s)+a t+b s}, \\
\text { where } a=\frac{\rho_{0 n 0}}{\rho_{1 n-10}}, b=\frac{\rho_{0 n-11}}{\rho_{1 n-10}} \text { and } \rho_{i j k} & =\frac{\omega_{i j k}^{*}}{\omega_{i j k}} .
\end{aligned}
$$


Proof Writing $P=\pi(\mathrm{t} \otimes \mathrm{s}) T_{n} \omega^{*}$ and $Q=\pi(\phi \otimes \mu) T_{n} \omega$ we have, from (2) and (4), that:

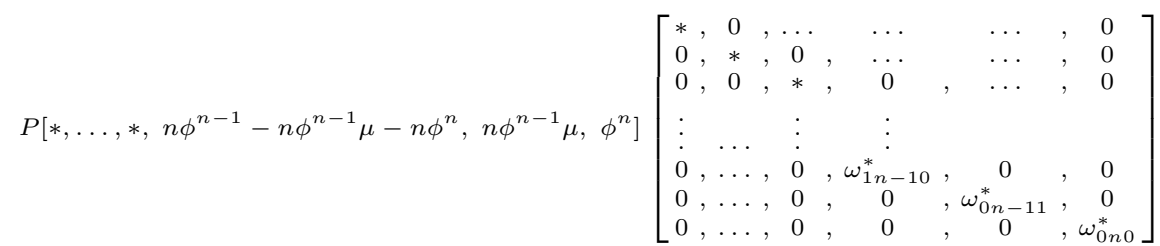

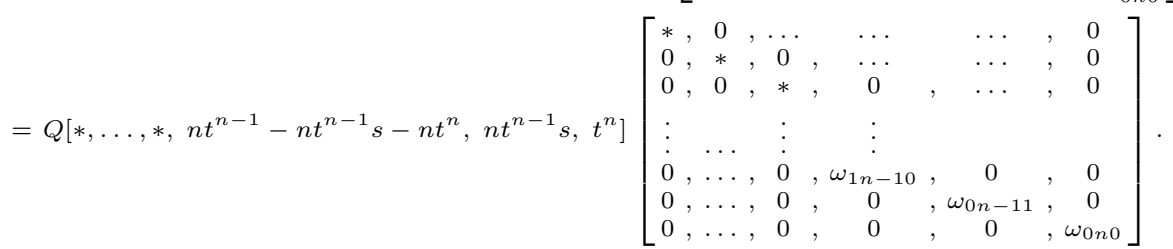

Hence

$$
\begin{aligned}
P \omega_{1 n-10}\left(n \phi^{n-1}-n \phi^{n-1} \mu-n \phi^{n}\right) & =Q \omega_{1 n-10}^{*}\left(n t^{n-1}-n t^{n-1} s-n t^{n}\right) \\
P \omega_{0 n-11} n \phi^{n-1} \mu & =Q \omega_{0 n-11}^{*} n t^{n-1} s \\
P \omega_{0 n 0} \phi^{n} & =Q \omega_{0 n 0}^{*} t^{n}
\end{aligned}
$$

from which we obtain, from $(5) /(6)$, that

$$
\frac{\omega_{0 n-11} \mu}{\omega_{0 n 0} \phi}=\frac{\omega_{0 n-11}^{*} s}{\omega_{0 n 0}^{*} t}
$$

i.e.,

$$
\phi=\left(\frac{\omega_{0 n-11}}{\omega_{0 n-11}^{*}}\right)\left(\frac{\omega_{0 n 0}}{\omega_{0 n 0}^{*}}\right)\left(\frac{t}{s}\right) \mu
$$

and from $(4) /(5)$ that

$$
\frac{\omega_{1 n-10}(1-\mu-\phi)}{\omega_{0 n-11} \mu}=\frac{\omega_{1 n-10}^{*}(1-s-t)}{\omega_{0 n-11}^{*} s}
$$

substituting for $\phi$, from (8), into (9), and solving for $\mu$ gives

$$
\mu(t, s)=\frac{\left(\frac{\omega_{0 n-11}^{*}}{\omega_{0 n-11}}\right) s}{\left(\frac{\omega_{1 n-10}^{*}}{\omega_{1 n-10}}\right)+s\left(\frac{\omega_{0 n-11}^{*}}{\omega_{0 n-11}}-\frac{\omega_{1 n-10}^{*}}{\omega_{1 n-10}}\right)+t\left(\frac{\omega_{0 n 0}^{*}}{\omega_{0 n 0}}-\frac{\omega_{1 n-10}^{*}}{\omega_{1 n-10}}\right)} ;
$$

and (8) then gives

$$
\phi(t, s)=\frac{\left(\frac{\omega_{0 n 0}^{*}}{\omega_{0 n 0}}\right) t}{\left(\frac{\omega_{1 n-10}^{*}}{\omega_{1 n-10}}\right)+s\left(\frac{\omega_{0 n-11}^{*}}{\omega_{0 n-11}}-\frac{\omega_{1 n-10}^{*}}{\omega_{1 n-10}}\right)+t\left(\frac{\omega_{0 n 0}^{*}}{\omega_{0 n 0}}-\frac{\omega_{1 n-10}^{*}}{\omega_{1 n-10}}\right)} .
$$

Writing $\rho_{i j k}=\frac{\omega_{i j k}^{*}}{\omega_{i j k}}$ we obtain

$$
\phi(t, s)=\frac{\rho_{0 n 0} t}{\rho_{1 n-10}+s\left(\rho_{0 n-11}-\rho_{1 n-10}\right)+t\left(\rho_{0 n 0}-\rho_{1 n-10}\right)}
$$


and

$$
\mu(t, s)=\frac{\rho_{0 n-11} s}{\rho_{1 n-10}+s\left(\rho_{0 n-11}-\rho_{1 n-10}\right)+t\left(\rho_{0 n 0}-\rho_{1 n-10}\right)} .
$$

The result now follows by writing $a=\frac{\rho_{0 n 0}}{\rho_{1 n-10}}$ and $b=\frac{\rho_{0 n-11}}{\rho_{1 n-10}}$.

It may be shown if $\phi$ and $\mu$ are as above and $\psi=(\phi, \mu)$ then $\psi$ maps $\Delta$ onto $\Delta$ in a continuous one-to-one manner if and only if $a>0$ and $b>0$; hence the only re-parametrisation, or invariant-image, solutions of (3) are those for which $a>0$ and $b>0$.

Using the form of Bézier triangles in relation (1) we obtain, from functional equation (3) and the $\phi, \mu$ functions of Lemma 1, that:

$$
\frac{\sum_{i+j+k=n} \frac{n !}{i ! j ! k !}(1-t-s)^{i} t^{j} s^{k} \omega_{i j k}^{*} v_{i j k}}{\sum_{i+j+k=n} \frac{n !}{i ! j ! k !}(1-t-s)^{i} t^{j} s^{k} \omega_{i j k}^{*}}=\frac{\sum_{i+j+k=n} \frac{n !}{i ! j ! k !}(1-t-s)^{i} t^{j} s^{k} a^{j} b^{k} \omega_{i j k} v_{i j k}}{\sum_{i+j+k=n} \frac{n !}{i ! j ! k !}(1-t-s)^{i} t^{j} s^{k} a^{j} b^{k} \omega_{i j k}}
$$

It follows that $\omega_{i j k}^{*}=c a^{j} b^{k} \omega_{i j k}$, where $c$ is an arbitrary non-zero multiplier. The following is therefore true:

Proposition 1 The functions $\tau_{B}\left[v, \omega^{*}\right]$ and $\tau_{B}[v, \omega]$ parametrise the same image for all $v$ :

- if an only if there exists a re-parametrisation function $\psi: \Delta \rightarrow \Delta$ such that $\tau_{B}\left[v, \omega^{*}\right]=\tau_{B}[v, \omega] \circ \psi$,

- if and only if $\psi=(\phi, \mu)$ where $\phi$ and $\mu$ are as in Lemma 1 with $a>0$ and $b>0$,

- if and only if

$$
\omega^{*}=c \operatorname{diag}\left(1, b, \ldots, b^{n}, a, a b, \ldots, a b^{n-1}, \ldots, a^{n-1} b, a^{n}\right) \omega
$$

for $a>0, b>0$ and $c \neq 0$.

The equivalence of re-parametrisation by the 2-variable Möbius transformations of Lemma 1 and the weight transformations defined by $\omega_{i j k}^{*}=c a^{j} b^{k} \omega_{i j k}$ has been observed previously (see [9], for example). Proposition 1 demonstrates that these Möbius functions are the only re-parametrisation solutions of the functional equation and, consequently, that $\omega_{i j k}^{*}=c a^{j} b^{k} \omega_{i j k}$ are the only transformations of the weights that are image-preserving for the Bézier triangles.

If $\mathbb{R}^{*}=\mathbb{R} \backslash\{0\}$ and $\mathbb{R}^{+}=\{x \in \mathbb{R}: x>0\}$, then the transformations defined by (10) determine a 3 -dimensional Lie transformation group $G$, isomorphic to $\mathbb{R}^{*} \times \mathbb{R}^{+} \times \mathbb{R}^{+}$ and parametrised by $a, b$ and $c$, acting on the $\frac{1}{2}(n+1)(n+2)$-dimensional manifold $\mathcal{O}_{n}$. In group-theory terms Proposition 1 states that: for a given vertex set $v$, the weight vectors $\omega^{*}$ and $\omega$ determine the same image under $\tau_{B}$ if and only if they lie on the same orbit of $G$ in $\mathcal{O}_{n}$.

A corollary of Proposition 1 is that, in general, a maximum of three weights can be transformed to unit modulus in any standardised representation of the triangles. 


\section{The Invariant functions of the degree- $n$ triangles}

5.1 The fundamental theorem on invariants

The action of a Lie group on a manifold is said to be semi-regular if all the orbits have the same dimension; as the stability subgroup of the $G$ action on $\mathcal{O}_{n}$ is trivial at each point of $\omega \in \mathcal{O}_{n}$, it follows that the orbits are each of dimension 3 and that the action is semi-regular. A fundamental theorem on the invariants of Lie group actions (see [10]) relates to the existence of functionally independent real-valued invariant functions on the manifold. In the case of semi-regular actions the theorem states, in essence, that:

'If $G$ is a Lie group acting semi-regularly on an $k$-dimensional manifold $X$ with $q$ dimensional orbits, then for each point $x \in X$ there exist $k-q$, real-valued functions $\iota_{1}, \ldots, \iota_{k-q}$ that are invariant and functionally-independent in some neighbourhood $N_{x}$ of $x$. Any other invariant $\iota$, defined on $N_{x}$, may be uniquely expressed as $\iota=$ $F\left(\iota_{1}, \ldots, \iota_{k-q}\right)$ for some $F$.'

As the orbits of $G$ in $\mathcal{O}_{n}$ have dimension 3, and $\mathcal{O}_{n}$ has dimension $\frac{1}{2}(n+1)(n+2)$ we have:

Corollary 1 At each point $\omega \in \mathcal{O}_{n}$ there are

$$
\frac{1}{2}(n+1)(n+2)-3
$$

functionally-independent, real-valued functions invariant under the action of $G$.

The primary aim of the remainder of the paper is to show that condition (10), of Proposition 1, for invariant image is equivalent to a simple condition expressed in terms of the invariant functions of $G$ on $\mathcal{O}_{n}$.

A set of invariant functions $I_{1}, \ldots, I_{p}$, real-valued or otherwise, is said to be complete for the action of $G$ on $X$ if their values uniquely identify orbits in the following sense: $x, x^{*} \in X$ lie on the same orbit of $G$ if and only if $I_{1}(x)=I_{1}\left(x^{*}\right), \ldots, I_{Q}(x)=$ $I_{Q}\left(x^{*}\right)$.The fundamental theorem does not provide the means to compute forms for invariant functions postulated; this problem is addressed below, for the $G$ action on $\mathcal{O}_{n}$, and complete sets of invariants are identified.

5.2 The invariant functions of the rational Bézier triangles

The first invariant identified below is not of the type covered by the fundamental theorem. It is discrete-valued, and shown later to be the invariant, additional to those of the fundamental theorem, required for a complete set.

Definition 1 We say that $\omega, \omega^{*} \in \mathcal{O}_{n}$ have the same sign-pattern, and write $\operatorname{sp}(\omega)=$ $s p\left(\omega^{*}\right)$, if:

either $\omega_{i j k}$ and $\omega_{i j k}^{*}$ have the same sign for all $0 \leq i, j, k \leq n$,

or $\omega_{i j k}$ and $-\omega_{i j k}^{*}$ have the same sign for all $0 \leq i, j, k \leq n$.

Observation $1 \mathrm{sp}$ is an invariant of the action of $G$ on $\mathcal{O}_{n}$. Equivalently, if $\omega$ and $\omega^{*}$ lie on the same orbit of $G$ then $\operatorname{sp}(\omega)=\operatorname{sp}\left(\omega^{*}\right)$. 
This follows immediately from (10), as $a>0, b>0$ and $c \in \mathbb{R} \backslash\{0\}$.

Further, a number of real-valued invariants may be computed from the following observation: if $A$ is a diagonal matrix and $x$ a column vector then $x^{*}=A x$ if and only if $\operatorname{diag}\left(x^{*}\right)=A \operatorname{diag}(x)$. It follows that the action of $G$ (relation (10)) is equivalent to $\Omega_{n}^{*}=c \operatorname{diag}\left(1, b, \ldots, b^{n}, a, a b, \ldots, a b^{n-1}, \ldots, a^{n-1} b, a^{n}\right) \Omega_{n}$ or

$$
\frac{1}{c} I_{n}=\operatorname{diag}\left(1, b, b^{2}, \ldots, b^{n}, a, a b, \ldots, a b^{n-1}, \ldots, a^{n-1}, a^{n-1} b, a^{n}\right) \Omega_{n} \Omega_{n}^{*-1}
$$

where $I_{n}$ is the $n \times n$ identity matrix, $a=\frac{\rho_{0 n 0}}{\rho_{1 n-10}}$ and $b=\frac{\rho_{0 n-11}}{\rho_{1 n-10}}$. As the left-hand side of (11) is a multiple of the identity, it follows that the elements of the diagonal matrix on the right-hand side are all equal. These equalities produce, following a little algebra, the $\frac{1}{2}(n+1)(n+2)-3$ invariant functions

$$
\varpi_{i j k}(\omega)=\frac{\omega_{i j k}}{\omega_{n 00}} \frac{\left(\omega_{1 n-10}\right)^{j+k}}{\left(\omega_{0 n 0}\right)^{j}\left(\omega_{0 n-11}\right)^{k}},
$$

for $i+j+k=n$ and $(i, j, k)$ not in $\{(n, 0,0),(0, n-1,0),(0, n, 0)\}$.

Lemma 2 The $\frac{1}{2}(n+1)(n+2)-3$ invariants, defined by (12):

1. are functionally independent,

2. are incomplete for the degree-n triangles.

Proof (1) Functional independence: the first $\frac{1}{2}(n+1)(n+2)-4$ invariant functions, $\varpi_{n-101}, \ldots, \varpi_{0 n-22}$ are all functions of a variable, namely $\omega_{i j k}$, that does not occur in any of the other functions of (12), and the last invariant, namely $\varpi_{1 n-10}$, is not a function of any of the variables unique to the others. It follows that the $\frac{1}{2}(n+1)(n+2)-3$ invariants of (12) are functionally independent (see Appendix).

(2) Incompleteness: let $\omega$ be such that $\omega_{i j k}>0$ and $\omega^{*}$ be the weight vector obtained from $\omega$ by reversing the signs of the $\omega_{1 n-10}, \omega_{0 n 0}$ and $\omega_{0 n-10}$. Clearly $s p\left(\omega^{*}\right) \neq s p(\omega)$ and, since $s p$ is an invariant under $G$, it follows that $\omega$ and $\omega^{*}$ do not lie on the same orbit. But $\varpi_{i j k}\left(\omega^{*}\right)=\varpi_{i j k}(\omega)$ for all $(i, j, k)$, hence there exist orbits of $G$ that are not distinguished by the invariant functions of (12) and they are incomplete.

Example 1 For the degree-2 triangles we have, from (11), the 5 relationships:

$$
\begin{aligned}
& \frac{1}{\rho_{200}}=\left(\frac{\rho_{011}}{\rho_{110}}\right) \frac{1}{\rho_{101}}, \frac{1}{\rho_{200}}=\left(\frac{\rho_{011}}{\rho_{110}}\right)^{2} \frac{1}{\rho_{002}}, \\
& \frac{1}{\rho_{200}}=\left(\frac{\rho_{020}}{\rho_{110}}\right) \frac{1}{\rho_{110}}, \frac{1}{\rho_{200}}=\left(\frac{\rho_{020}}{\rho_{110}}\right)\left(\frac{\rho_{011}}{\rho_{110}}\right) \frac{1}{\rho_{011}}, \\
& \frac{1}{\rho_{200}}=\left(\frac{\rho_{020}}{\rho_{110}}\right)^{2} \frac{1}{\rho_{020}}
\end{aligned}
$$

the first three of which give, respectively, the invariant functions

$$
\varpi_{101}(\omega)=\frac{\omega_{101}}{\omega_{200}}\left(\frac{\omega_{110}}{\omega_{011}}\right), \varpi_{002}(\omega)=\frac{\omega_{002}}{\omega_{200}}\left(\frac{\omega_{110}}{\omega_{011}}\right)^{2}, \varpi_{110}(\omega)=\frac{\omega_{110}}{\omega_{200}}\left(\frac{\omega_{110}}{\omega_{020}}\right) .
$$

The final two relations both give invariants identical to $\varpi_{110}$. The invariants may be expressed in the form

$$
\varpi_{i j k}(\omega)=\frac{\omega_{i j k}}{\omega_{200}} \frac{\left(\omega_{110}\right)^{j+k}}{\left(\omega_{020}\right)^{j}\left(\omega_{011}\right)^{k}}
$$


where $i, j, k \in\{0,1,2\}$, with $i+j+k=2$, except for $(2,0,0),(0,1,1)$ and $(0,2,0)$. The variable $\omega_{101}$ is unique to $\varpi_{101}, \omega_{002}$ is unique to $\varpi_{002}$ and $\varpi_{110}$ is not a function of either $\omega_{101}$ or $\omega_{002}$; the three invariants are therefore independent.

The next result demonstrates that the $\varpi_{i j k}$ functions of (12) and the sign-pattern invariant $s p$ determine a complete set of invariants for the action of $G$ on $\mathcal{O}_{n}$.

Theorem 1 Two weight vectors $\omega, \omega^{*} \in \mathcal{O}_{n}$ are such that $\omega_{i j k}^{*}=c a^{j} b^{k} \omega_{i j k}$, for some $c \in \mathbb{R} \backslash\{0\}, a>0$ and $b>0$, if and only if

$$
s p(\omega)=s p\left(\omega^{*}\right) \text { and } \varpi_{i j k}(\omega)=\varpi_{i j k}\left(\omega^{*}\right) .
$$

Proof (i) If $\omega_{i j k}^{*}=c a^{j} b^{k} \omega_{i j k}$, for some $c \in \mathbb{R} \backslash\{0\}, a>0$ and $b>0$ then the relations $s p(\omega)=s p\left(\omega^{*}\right)$ and $\varpi_{i j k}(\omega)=\varpi_{i j k}\left(\omega^{*}\right)$ follow directly by substitution.

(ii) If $s p(\omega)=s p\left(\omega^{*}\right)$ and $\varpi_{i j k}(\omega)=\varpi_{i j k}\left(\omega^{*}\right)$ then defining $\hat{c}=\frac{\omega_{n 00}^{*}}{\omega_{n 00}}, \hat{a}=$ $\frac{\omega_{1 n-10} \omega_{0 n 0}^{*}}{\omega_{1 n-10}^{*} \omega_{0 n 0}}$ and $\hat{b}=\frac{\omega_{1 n-10} \omega_{0 n-11}^{*}}{\omega_{0 n-11}^{*} \omega_{0 n-11}}$ it follows that if $\omega_{i j k}$ and $\omega_{i j k}^{*}$ have the the same sign then $\hat{c}, \hat{a}, \hat{b}>0$, and that if $\omega_{i j k}$ and $\omega_{i j k}^{*}$ are of opposite sign then $\hat{c}<0$, and $\hat{a}, \hat{b}>0$. Further:

$$
\begin{aligned}
\hat{c} \hat{a}^{j} \hat{b}^{k} \omega_{i j k} & =\frac{\varpi_{i j k}(\omega)}{\varpi_{i j k}\left(\omega^{*}\right)} \omega_{i j k}^{*}, \\
& =\omega_{i j k}^{*} .
\end{aligned}
$$

which completes the proof.

We now have:

Proposition 2 The functions $\tau_{B}\left[v, \omega^{*}\right]$ and $\tau_{B}[v, \omega]$ parametrise the same image for all $v$ if and only if

$$
s p\left(\omega^{*}\right)=s p(\omega) \text { and } \varpi_{i j k}\left(\omega^{*}\right)=\varpi_{i j k}(\omega)
$$

for all $i, j, k \in\{0,1, \ldots, n\}$ with $i+j+k=n$ except $(n, 0,0),(0, n-1,1)$ and $(0, n, 0)$.

Alternative methods exist for the determination of invariants for the Bézier triangles. For example, the group $G$ allows certain triples of components of $\omega$ to be transformed to unit modulus, the remaining components being transformed to independent invariant functions. The general context of this 'normalisation' process is described by Olver in [11], and may be applied to the Bézier triangles to yield, for example, the $\frac{1}{2}(n+1)(n+$ 2) -3 real-valued, independent functions defined by:

$$
\varpi_{i j k}^{*}=\omega_{i j k} \frac{\left(\omega_{n 00}\right)^{j+k-1}}{\left(\omega_{n-110}\right)^{j}\left(\omega_{n-101}\right)^{k}}
$$

which may be substituted, in the above results, for the $\varpi_{i j k}$ functions. 
Appendix

A. Functional independence

Definition 2 (see [10]) A set $\left\{f_{1}, \ldots, f_{k}\right\}$ of smooth real-valued functions on a manifold $M$ having a common domain of definition is said to be functionally independent if for each $x_{0} \in M$ there is a neighbourhood $U$ and a smooth function $H\left(z_{1}, \ldots, z_{k}\right)$, not identically zero on any subset of $\mathbb{R}^{k}$, such that $H\left(f_{1}(x), \ldots, f_{k}(x)\right)=0$ for all $x \in U$. The functions are called functionally independent if they are not functionally dependent when restricted to any open subset of $M$.

In the following situations functional independence is clear:

1. if each $f_{i}$ is a function of a variable that does not occur in the other functions,

2. if all except one of $f_{1}, \ldots, f_{k}$, is a function of a variable that does not occur in the other functions, and the exceptional function does not depend on any of the variables unique to the others.

B. Structure matrices for the low-degree Bézier triangles

The structure matrices for the Bézier triangles of up to degree 3 are, respectively:

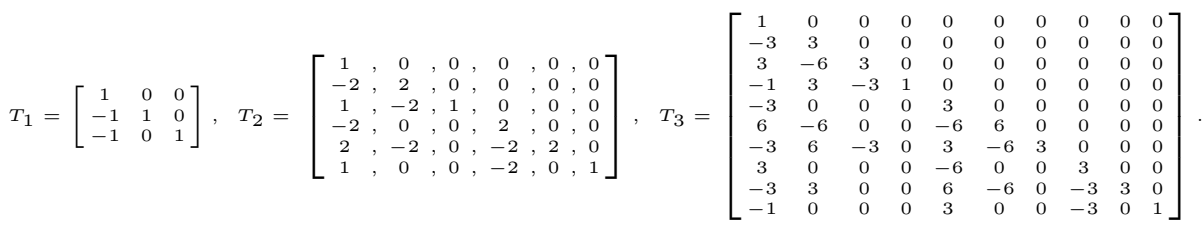

Acknowledgements The author would like to thank Irina Kogan for identifying a number of imprecise statements and presentational inefficiencies in the original version of this paper. As a result significant changes have been made to section 5, which have improved the quality of this paper.

\section{References}

1. Patterson, R.R., Projective transformations of the parameter of a Bernstein-Bézier curve, ACM Trans. on Graphics 4(4), 276-290, (1985).

2. Farin, G., NURBS from Projective Geometry to Practical Use, Second Edition, A.K.Peters, Massachusetts, (1999).

3. Zheng, J., Minimizing the maximal ratio of weights of a rational Bézier curve, Computer Aided Geometric Design, 22(3), 275-280, (2005).

4. Cai, H.J. \& Wang, G.J., Minimizing the maximal ratio of weights of rational Bézier curves and surfaces, Computer Aided Geometric Design, (to appear).

5. Bez, H.E., Invariant-geometry conditions for the rational bi-quadratic Bézier surfaces, Computer Aided Geometric Design, 26(8), 877-887, (2009).

6. Bez, H.E., The invariant functions of the rational bi-cubic Bézier surfaces, Lecture Notes in Computer Science 5654, 55-74, (2009). 
7. Gallier, J. (1999), A simple method for drawing a rational curve as two Bézier segments, ACM Transactions on Graphics, 18(4), 316-328, (1999).

8. Gallier, J., Simple methods for drawing rational surfaces as four or six Bézier patches, arXiv:cs/0606055v1 [cs:CG] 12 June 2006.

9. Joe, B. \& Wang, W., Reparametrization of rational triangular Bézier surfaces, Computer Aided Geometric Design, 11(4), 354-361, (1994).

10. Olver, P.J., Equivalence, Invariants, and Symmetry, Cambridge University Press, Cambridge, (1995).

11. Olver, P.J., Classical Invariant Theory, London Mathematical Society Student Texts, 44, Cambridge University Press, Cambridge, (1999). 